\title{
Surgical Management of Optic Disc Pit Maculopathy with Outer Retinal Hole
}

\author{
Aditya Shreekant Kelkar Jai Aditya Kelkar \\ Devanshi Ramji Bhanushali Shreekant Bhaskar Kelkar
}

National Institute of Ophthalmology, Pune, India

Key Words

Optic pit · Outer retinal hole $\cdot$ Retinoschisis · Vitrectomy

\begin{abstract}
Objective: To report the successful outcome of a rare optic pit-associated maculopathy with an outer retinal hole following $23 \mathrm{G}$ vitrectomy, internal limiting membrane (ILM) peeling and fluid-gas exchange without additional endolaser.

Method: Interventional case report.

Results: This case report documents a 56-year-old male patient with complaints of progressive diminution of vision in the right eye more than in the left eye due to an optic disc pit with an outer retinal hole and a cataract. Optical coherence tomography confirmed the presence of an outer retinal hole. The case report shows the successful outcome of a rare optic pit-associated maculopathy with an outer retinal hole and a cataract following phacoemulsification with $23 \mathrm{G}$ vitrectomy, ILM peeling and fluid-gas exchange without additional endolaser.
\end{abstract}

\section{Introduction}

Optic pits occur equally between men and women [1]. They are seen in roughly 1 in 10,000 eyes, and approximately $85 \%$ of optic pits are found to be unilateral. Macular holes complicating optic pits were described first by Reis in 1908 [2]. Optic pits have been associated with serous retinal detachments $[3,4]$ in up to as many as $50 \%$ of all cases. An outer layer hole appears to be uncommon in optic pit maculopathy. Imamura et al. [5] reported an outer layer hole in only $27 \%$ of eyes with retinal detachment associated with optic disc maculopathy. The detachments associated with optic pits may occur at any age but most frequently present in early adulthood. The most popular theory behind this association is a separation of the layers of the retina, known as retinoschisis, due to fluid (the vitreous humor) entering the optic pit and traveling 
between the inner and outer layers of the retina. The outer layer may then subsequently detach.

We hereby present a case report showing successful management of a rare optic disc pit anomaly with an outer retinal hole and a cataract, which was treated with phacoemulsification vitrectomy with gas tamponade but without endolaser. Marked improvement in visual acuity corresponded to the resolution of the outer retinal hole observed clinically as well as on optical coherence tomography (OCT).

\section{Case Report}

A 56-year-old male presented with a 6-month history of painless, progressive diminution of vision in the right eye more than in the left eye. His past medical history and ocular history were not significant, and there was no history of trauma or surgery.

Ophthalmic examination showed the best-corrected visual acuity of counting fingers 2 meters in the right eye and 20/40 in the left eye. On anterior segment examination, both pupils reacted normally to light, whereas slit-lamp biomicroscopy revealed grade II nuclear sclerosis in both eyes more so in the right than in the left. The intraocular pressure was normal in both eyes.

Dilated fundus examination of the right eye revealed an oval, gray-yellow crater-like depression on the temporal aspect of the optic disc, suggestive of an optic pit (fig. 1). In the macular area adjoining the disc there was a serous elevation of around 4 disc diameters with central thinning of the retinal layers, giving the impression of an outer retinal hole. OCT was done and confirmed the presence of an optic pit with maculopathy and an outer retinal hole (fig. 2). Fundus fluorescein angiography showed a window defect corresponding to the outer retinal hole in addition to pooling of the dye in the late venous phase, suggestive of serous retinal detachment ( $\underline{\text { fig. } 3}$ ).

Pros and cons of various treatment options including no treatment were discussed. After obtaining informed consent, the patient underwent phacoemulsification with intraocular lens implantation with $23 \mathrm{G}$ pars plana vitrectomy with SF6 gas tamponade without additional endolaser.

The vitreous was stained with triamcinolone $0.1 \mathrm{ml}$, and the induction of posterior vitreous detachment was achieved with the vitreous cutter. After completing the vitrectomy, internal limiting membrane (ILM) peeling was done from arcade to arcade including the disc margin using ILM peeling forceps. The periphery was scanned for breaks and no offensive lesions were noted. After fluid air exchange, 20\% SF6 air-gas mixture was injected. The cannulas were removed and no leakage was noted from the cannula sites. The intraocular pressure was digitally normal at the end of the procedure. The patient was instructed to maintain a face-down position for 1 week and was followed up closely. After 2 weeks, the gas bubble was completely absorbed, and vision had improved to 20/60.

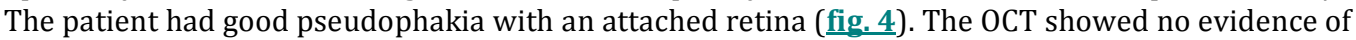
serous retinal detachment or retinoschisis, and the outer retinal break was sealed (fig. 5). The patient was followed up for the next 2 years on a regular basis and there was no evidence of recurrence. The patient maintained $20 / 60$ vision in all follow-ups.

\section{Discussion}

This case report documents the successful outcome of a rare optic pit-associated maculopathy with an outer retinal hole and a cataract following phacoemulsification 23 G vitrectomy, ILM peeling and fluid-gas exchange without additional endolaser.

An outer layer hole appears to be uncommon in optic pit maculopathy. Imamura et al. [5] reported an outer layer hole in only $27 \%$ of eyes with retinal detachment associated with optic disc maculopathy. 
The treatment options include focal laser to the temporal aspect of the optic pit. However, a relatively high incidence of recurrence [6] has been reported after laser treatment alone; laser treatment also results in temporal scotoma to the patient [7]. Hirakata et al. [8] reported the success of vitrectomy with induction of posterior vitreous detachment and gas tamponade, without additional laser treatment in reattaching the macula, and improvement in central vision in most patients with optic disc pit maculopathy.

In our patient, the macular schisis resolved and the outer retinal hole sealed after surgical intervention. There was no recurrence of maculopathy at the 2-year follow-up. His visual acuity remained at 20/60 at the end of 2 years of follow-up. The limited recovery in visual function is probably related to the longstanding nature of the condition.

Vitrectomy with ILM peeling and gas tamponade without any additional laser photocoagulation may be sufficient for the treatment of optic disc pit maculopathy even with an outer retinal hole. Although further studies are required to evaluate the above findings, documenting large series is challenging due to the rarity of cases with optic disc maculopathy with outer retinal holes.

\section{Disclosure Statement}

None of the authors has a financial or proprietary interest in any material or method mentioned.

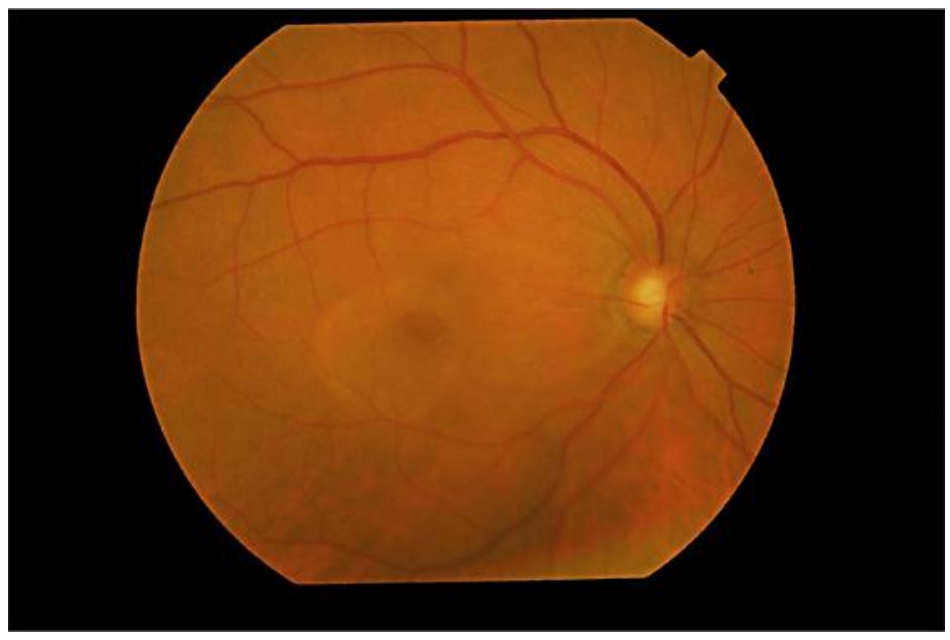

Fig. 1. Fundus examination of the right eye revealing an oval, gray-yellow crater-like depression on the temporal aspect of the optic disc, suggestive of an optic pit. 


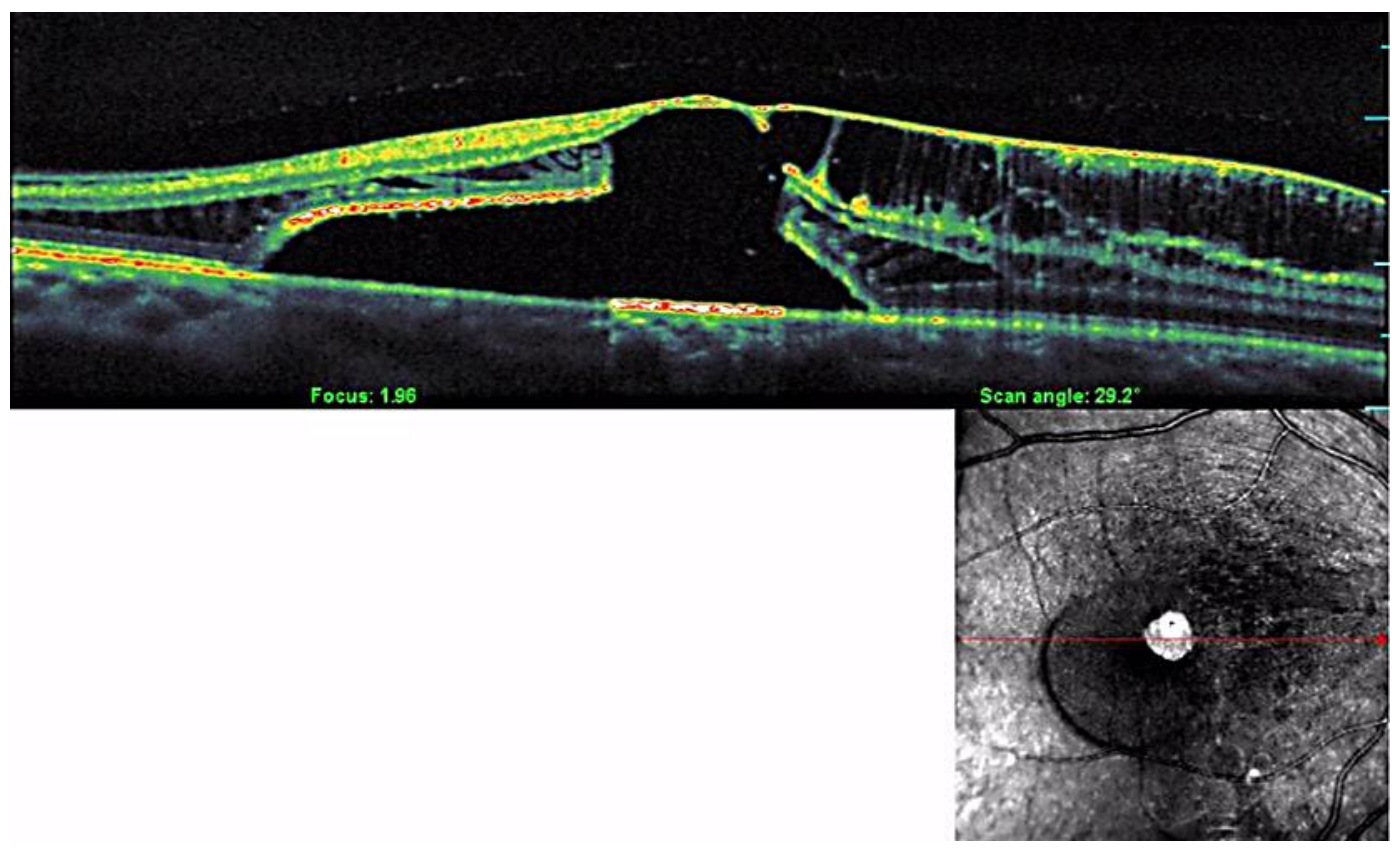

Fig. 2. OCT confirming the presence of an optic pit with maculopathy and an outer retinal break.

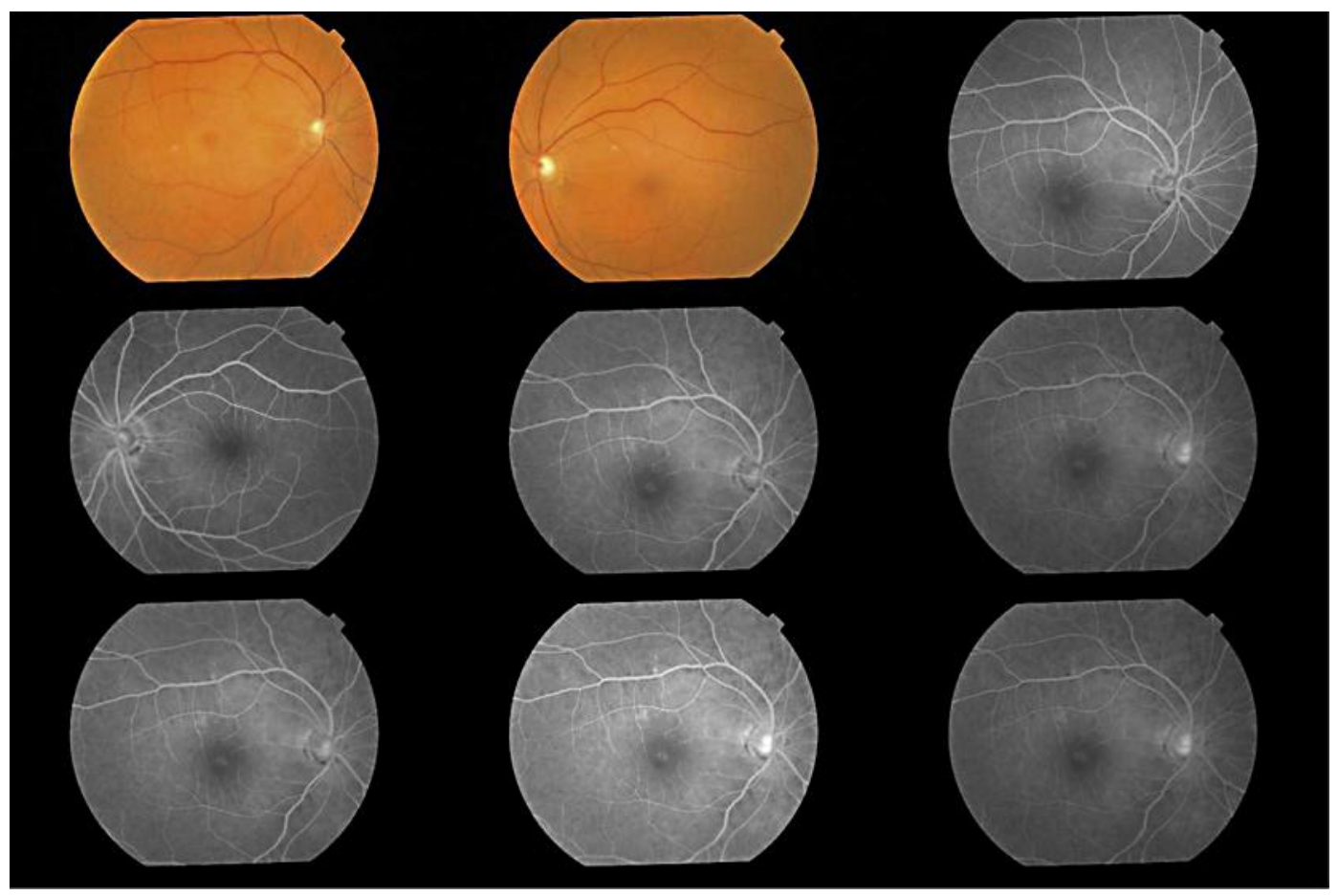

Fig. 3. Fundus fluorescein angiography showing a window defect corresponding to the outer retinal hole in addition to pooling of the dye in the late venous phase, suggestive of serous retinal detachment. 


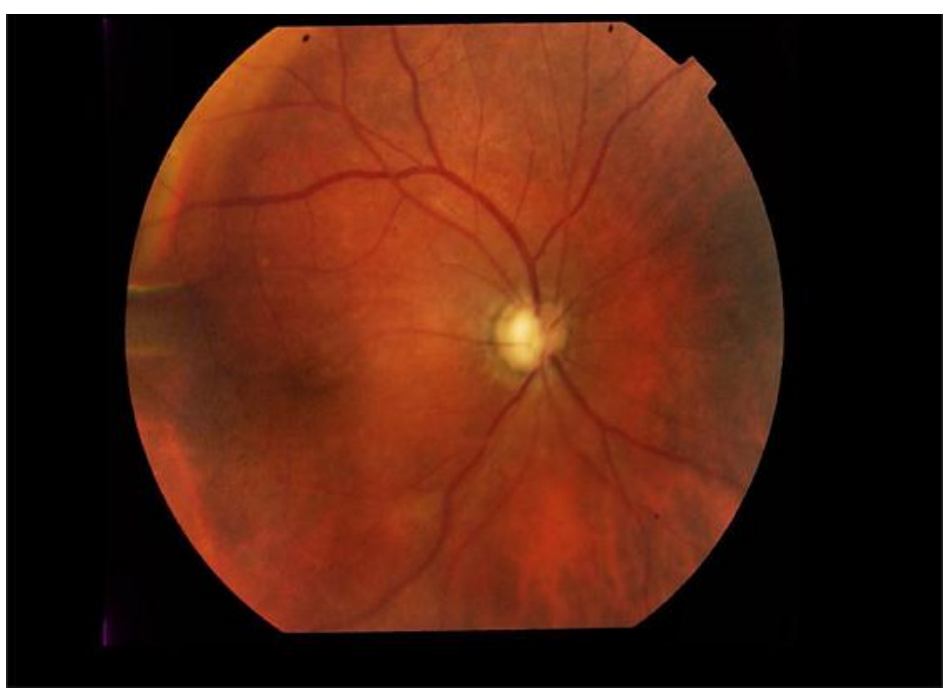

Fig. 4. Fundus photograph of the right eye showing a resolved outer retinal hole with attached retina, postoperatively.

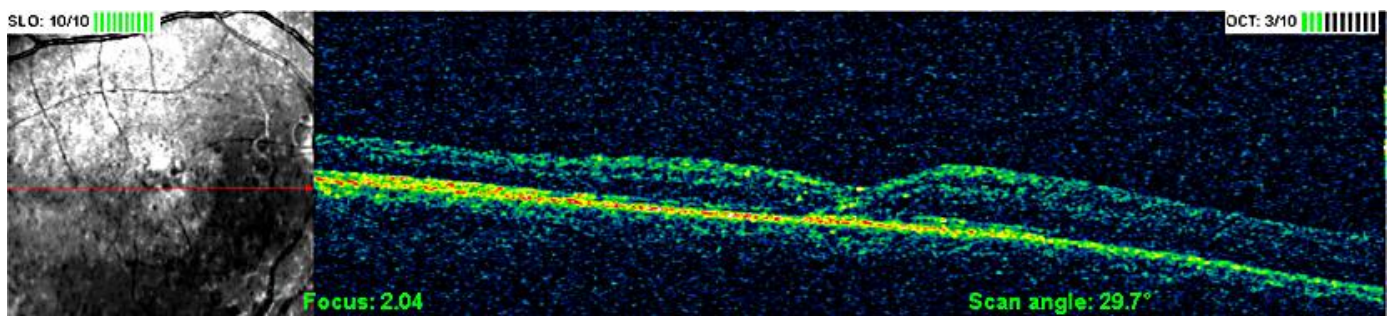

Fig. 5. OCT showing no evidence of serous retinal detachment or retinoschisis, and the outer retinal break is sealed.

\section{References}

1 Wagner-Schuman M, Dubis AM, Nordgren RN, Lei Y, Odell D, Chiao H, Weh E, Fischer W, Sulai Y, Dubra A, Carroll J: Race- and sex-related differences in retinal thickness and foveal pit morphology. Invest Ophthalmol Vis Sci 2011;52:625-634.

2 Reis W: Eine wenig bekannte typische Missbildung am Sehnerveneintritt: umschriebene Grubenbildung auf der Papilla n. optici. Z Augenheilkd 1908;19:505-528.

3 Sadun AA: Optic disc pits and associated serous macular detachment; in Ryan SJ (ed): Retina, ed 4. St. Louis, Mosby, 2005, chapter 111.

-4 Ferry AP: Macular detachment associated with congenital pit of the optic nerve head. Arch Ophthalmol 1963;70:346-357.

5 Imamura Y, Zweifel SA, Fujiwara T, Freund KB, Spaide RF: High-resolution optical coherence tomography findings in optic pit maculopathy. Retina 2010;30:1104-1112.

-6 Postel EA, Pulido JS, Mc Namara JA, Johnson MW: The etiology and treatment of macular detachment associated with optic nerve pits and related anomalies. Trans Am Ophthalmol Soc 1998;96:73-88, discussion 88-93.

-7 Falcone PM, Lou PL: Resolution of an external layer macular hole associated with an optic nerve pit after laser photocoagulation. Br J Ophthalmol 1993;77:457-459.

-8 Hirakata A, Okada AA, Hida T: Long-term results of vitrectomy without laser treatment for macular detachment associated with an optic disc pit. Ophthalmology 2005;112:1430-1435. 